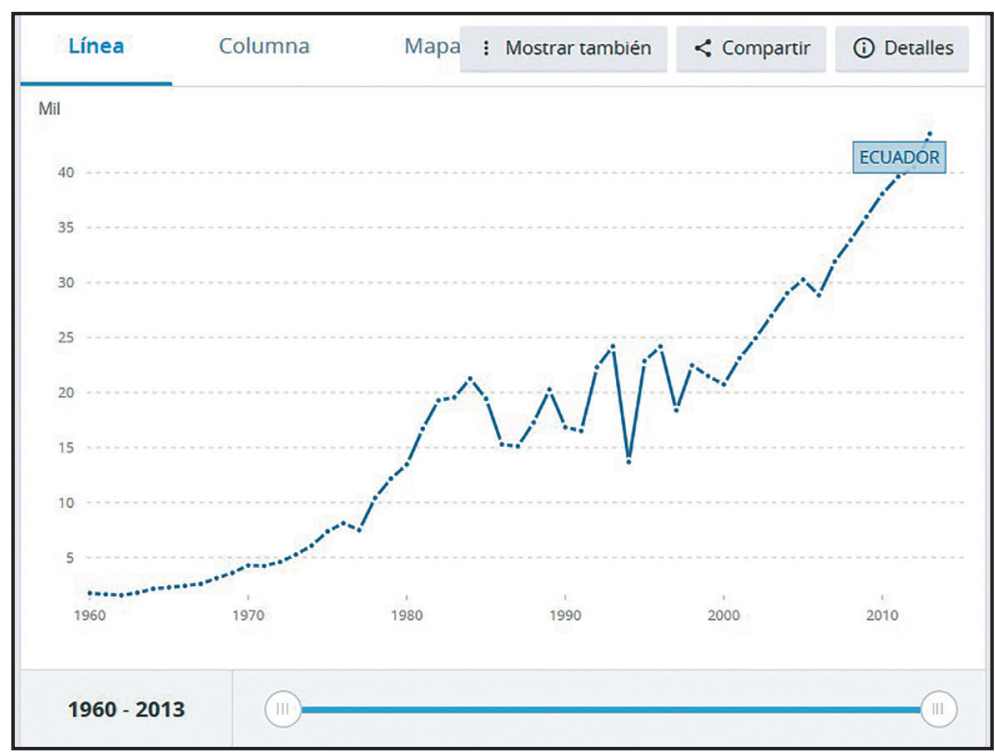

Emisiones de $\mathrm{CO} 2(\mathrm{kt})$ en Ecuador (1960-2013)

http://datos.bancomundial.org/indicador/EN.ATM.CO2E.KT?end=2013 \&locations $=E C \&$ start $=1960$ \&view $=$ chart

\title{
DEMOCRACIA, AMBIENTE Y ECOLOGÍA
}

Ansaloni, Raffaella

Correspondencia: ransaloni@uazuay.edu.ec 


\title{
Resumen
}

Las actividades humanas han provocado graves consecuencias sobre el ambiente, especialmente en los últimos dos siglos. Las principales causas que han llevado a la degradación de nuestra tierra son: la sobreexplotación de los recursos naturales, el escaso respeto y comprensión de los mecanismos naturales de autorregulación y las crecientes emisiones de gases de efecto invernadero, fruto del crecimiento demográfico y, sobre todo, de la globalización del mercado y del sistema económico basado en el consumismo. No se aprecia, hasta el momento, ningún cambio de ruta en la economía global, por eso es indispensable actuar para anteponer el interés colectivo al beneficio de algunos grupos de poder.

Palabras clave: Ambiente, cambio climático, recursos naturales, consumismo, globalización.

\section{DEMOCRACY, ENVIRONMENT AND ECOLOGY}

\begin{abstract}
Human activity has had serious consequences on the environment, especially in the last two centuries. The main causes that have led to the degradation of our land are the overexploitation of natural resources, little respect and understanding of the self-regulation natural mechanisms, growing greenhouse gas emissions due to population growth; and, above all, globalization of the market and of the economic system based on consumerism. No change of course in the global economy has been seen so far; therefore, it is essential to act to put the collective interest before the benefit of some groups of power.
\end{abstract}

Keywords: environment, climate change, natural resources, consumerism, globalization. 
Las tres palabras que me propusieron los editores de la revista Universidad-Verdad para desarrollar este artículo, me generaron una cierta incertidumbre. Democracia, ambiente y ecología...... ¡de cuántas maneras podemos aproximarnos a estos temas! Para una profana de las ciencias sociales como yo, resulta muy difícil enlazar la democracia con la preservación de nuestro ambiente y de la naturaleza.

El significado literario de la palabra democracia "gobierno del pueblo" nos engaña; actualmente hablamos de democracia representativa, democracia liberal, democracia popular y otros conceptos alejados de la voluntad del pueblo, de los ciudadanos. El New York Times reportó que las dos terceras partes de los estadounidenses quieren que EEUU se adhieran a los tratados internacionales vinculantes para limitar las emisiones de $\mathrm{CO}_{2}$, los mismos tratados que el parlamento estadounidense (de mayoría republicana) no ha ratificado. (Russonello, 2015). En otras palabras, los representantes electos no respetan la voluntad de sus electores, al menos en este campo de los problemas ambientales y del cambio climático. La democracia representativa y liberal de los Estados Unidos ha entonces fracasado en el cuidado ambiental global, ya que, con la negación de la existencia del cambio climático y la oposición a todo tipo de regulación de emisiones, "el partido republicano constituye un peligro real para la digna supervivencia de la especie humana". (Chomsky, 2016)

Sin embargo, algunos países industrializados y democráticos, como Alemania y otros países europeos, han hecho de la lucha contra el cambio climático su bandera, convirtiendo la casi totalidad de su matriz energética a las 
energías renovables. Entonces, en por lo menos uno de los problemas ambientales que más nos preocupan, el cambio climático, la forma de gobierno no determina las acciones que el Estado toma, e influyen otros factores, principalmente económicos y productivos, que aquí no analizaremos.

Al referirnos al "ambiente" estamos enfocando al ambiente biofísico o medio. El entorno biofísico incluye plantas, animales, microorganismos así como aire, clima, agua, suelo, y demás componentes. Las ciencias ambientales estudian las interacciones que ocurren en el ambiente y parte de esta disciplina científica se centra en los efectos de las actividades humanas sobre éste. Por otro lado, la ecología es una ciencia interdisciplinaria (incluye la biología, la biogeografía y la ciencia de la Tierra), que estudia las relaciones mutuas existentes entre los seres vivos y las que tienen con el medio en el cual viven (con los llamados "factores abióticos"). (Nebel \& Wright, 1999); (Laferriere \& Stoett, 2003).

Estas dos disciplinas, los estudios ambientales y la ecología, frecuentemente son asociadas con las palabras ambientalismo y ecologismo, y con los activistas que tienen como bandera la protección del ambiente, de la naturaleza, la biodiversidad, el agua, etc. Sin ser activistas, muchos de nosotros estamos atentos a nuestro entorno y tratamos de cuidar el ambiente, sobre todo ahora, cuando las actividades humanas repercuten gravemente en nuestras vidas. Desafortunadamente el esfuerzo individual es importante pero no es suficiente para revertir la degradación de nuestra "casa", que se ve afectada por presiones demográficas y económicas gigantescas. 
¿Cómo llegamos a este punto? ¿Podemos cambiar de ruta y vivir en armonía con nuestro entorno natural? Responder es difícil, pero vale la pena enfocar tres puntos clave, que pueden ayudarnos a aclarar parcialmente estas preguntas y a buscar respuestas.

El primer punto es que los recursos naturales son limitados y se agotan. Esta afirmación que parece tan obvia, en realidad no lo era hace un siglo o más. Y por esa razón los Estados no impulsaban acciones de protección hacia sus recursos. En el Ecuador, uno de los ejemplos más claros fue la sobreexplotación de Cinchona, la planta conocida como "cascarilla" o "quina", de la cual se extraía la quinina, potente remedio contra la malaria. Desde finales del siglo XVII y por más de dos siglos su recolección fue tan intensa que provocó la drástica reducción de las poblaciones, tanto así que durante la segunda guerra mundial, cuando se reactivó el mercado de esta planta, se tuvo que buscarla en lugares muy alejados y de difícil acceso, como por ejemplo la inhóspita (para aquel tiempo) zona de Molleturo. Ni durante la colonia, ni en época republicana, se tomaron acciones para preservar este importante recurso, que, además de los beneficios generados para la humanidad, sostuvo la economía de Cuenca durante dos siglos.

En la actualidad, la obvia afirmación tampoco es entendida cabalmente, porque si se tuviera conciencia de que el agua se agota, todos los municipios recolectarían y tratarían las aguas servidas. $Y$ las fuentes hídricas $y$ áreas aledañas serían intangibles: allí no podrían estar ni ganado, ni cultivos, ni plantaciones forestales ni mineras. Para preservar las fuentes de agua, debería intervenir el gobierno (estatal o local) "democrático": se respetaría la propiedad privada o comunal, pero cuando no afecta al 
derecho colectivo de tener agua. En este caso, ninguna persona o comunidad podría usar esas áreas, previa compensación económica, y la autoridad competente se encargaría de su preservación. O en alternativa, la conservación del área estaría a cargo del propietario (individual o colectivo), al cual se le reconocería un pago por este servicio. Asimismo, y privilegiando los intereses colectivos y la preservación de los recursos naturales, las autoridades no deberían otorgar ningún permiso de explotación minera en esta zona, pese a que esto afecte a los ingresos económicos estatales (o locales), que se recaudarían por concepto de impuestos.

El segundo punto es que nuestro entorno, la naturaleza,es un sistema complejo, en el cual todas las piezas, plantas, animales, microorganismos, clima, aire, agua, suelo, rocas, están relacionadas entre sí. Esto implica que es difícil prever la reacción de la naturaleza a nuestras acciones. Muchos ecólogos han estudiado estos fenómenos, el libro Gaia ha divulgado de manera científica estas teorías; incluso la película Avatar ha mostrado a todos, añadiendo mucha fantasía, las terribles consecuencias de nuestras acciones (Lovelock, 1992) (Cameron, 2009) Por largo tiempo y hasta nuestros días, el ser humano ha actuado prestando poca atención a estos complejos mecanismos, centrándose solo en el efecto inmediato, tanto que sólo después de décadas hemos entendido que los insecticidas afectan más a las ranas que a los mosquitos y que la excesiva iluminación nocturna espanta más a las aves que a los ladrones.

La naturaleza tiene propiedades intrínsecas de autorregulación, que hemos despreciado, es suficiente fijarse en el paradigma de la llamada Revolución Verde 
(que a propósito, fue impulsada para contrarrestar las revoluciones "rojas" y defender así la "democracia"), para la cual la fertilidad no es una propiedad del suelo, sino de los abonos químicos. De esta manera, hemos contaminado tanto el aire a través de la producción industrial de fertilizantes, como el suelo y el agua, distribuyendo estos mismos fertilizantes sin medida. La "madre tierra", concepto presente en todas las culturas campesinas, que proporciona alimento y abrigo, y que debe ser respetada, se transformó así en "tierra vacía" (Shiva, 1997), con un sinnúmero de impactos negativos sobre ella y el entero planeta.

El tercer punto es que el sistema económico basado en el consumismo y la globalización es incompatible con la preservación del ambiente y sus equilibrios. Pensemos en la cantidad de desechos que se producen al consumir más, a la energía que se emplea para producir lo que se consume, al combustible necesario para transportar de un lado al otro del planeta grandes cantidades de productos. No es una simple percepción: el último informe de las Naciones Unidas sobre los objetivos de desarrollo del milenio revela que las emisiones de $\mathrm{CO}_{2}$ se han incrementado en un $50 \%$ de 1990 a 2015, de manera creciente, debido a la deforestación y sobre todo al crecimiento económico de los países de Asia oriental, en los cuales la industria ha descentralizado su producción, sobre todo la más contaminante. (ONU, 2015).

Es fácil darnos cuenta de cuánto estamos consumiendo ahora con respecto a dos décadas atrás: No había envases plásticos (botellas y otros objetos desechables), que contaminan tanto para ser producidos como para ser destruidos. Teníamos muchas menos "cosas", objetos 
de los cuales, en muchos casos, podríamos prescindir. ¿Cuántas son las prendas de vestir que están en nuestro armario y que realmente usamos? ¿Cuántos equipos electrónicos en desuso tenemos? ¿Cuántos alimentos desperdiciamos en la cadena entre el productor y el consumidor? Los ejemplos son innumerables, y cada uno de nosotros puede elaborar largas listas de cosas inútiles que hemos adquirido, inducidos por cualquier motivo.

Los supermercados están llenos de productos de todo lado; hasta llegar a paradojas como que en Cuenca se venda agua embotellada proveniente de Italia y en Italia agua embotellada de Vilcabamba! Entre otras cosas, transportar el agua embotellada requiere de mucho gasto energético, debido a su peso específico. El transporte de todo a todo lado es realmente un problema ambiental enorme, así como lo es el transporte de las personas en vehículos particulares, en primer lugar por las emisiones generadas.

El sistema económico globalizado y basado en el consumo no tiene que ver con el sistema político: tanto las democracias liberales occidentales (Europa, Norteamérica) y del extremo oriente (Japón, Corea), como los Estados comunistas como China están dentro de este modelo. El creciente consumismo caracteriza también muchos países del tercer mundo, con la agravante de que en estos países no existe cuidado ambiental alguno. Pensemos, por ejemplo, que Indonesia y Vietnam son entre los países que más contaminan los mares con plástico. Podemos observar que tampoco el llamado"socialismo del siglo XXI" ha logrado cambiar este paradigma: basando el crecimiento económico en la explotación de los recursos naturales (petróleo y minerales), dejando que los ciudadanos tengan 
completa libertad de consumo, evitando penalizaciones fiscales para las industrias y actividades contaminantes.

El sistema económico mundial impone sus reglas, y pese a que las acciones que de ello derivan son negativas para nuestra tierra, ningún Estado las rompe, pese a que algunos países tienen prácticas ambientalmente más amigables que otros. En cambio, desde el punto de vista ecológico, vemos que los más eficaces métodos de protección ambiental son la desglobalización y la despetrolización. El fin de la globalización es visto en esta época como algo negativo; sin embargo, en términos ecológicos llevaría a incrementar el uso de productos locales y bajar el nivel de consumo, lo que se traduciría en menores gastos energéticos y menor contaminación. Por otro lado, el fin de la dependencia de los combustibles fósiles y en especial del petróleo, puede ser alcanzado transportando menos mercadería, favoreciendo el transporte colectivo de personas, empleando fuentes energéticas renovables, optimizando el uso de energía y produciendo sólo lo necesario. En una sociedad verdaderamente democrática, o sea bajo el gobierno de gente pensante y con sentimientos, no sería difícil alcanzar estos dos objetivos. Metas que, en cambio, son inalcanzables en este mundo gobernado por los mercados, en el cual estamos ocupados en consumir, más que pensar y sentir. 


\section{Bibliografía}

Cameron, J. (Dirección). (2009). Avatar [Película].

Chomsky, N. (2016). Who rules the world? New York: Henry Holt and Company.

Laferriere, E., \& Stoett, P. (2003). International Relations Theory and Ecological Thought: Towards a Synthesis. New York: Routledge.

Lovelock, J. (1992). Gaia, una ciencia para curar el planeta. Barcelona: Oasis S. L.

Nebel, B., \& Wright, R. (1999). Ciencias Ambientales. Ecología y desarrollo sostenible (6ta ed.). México: Prentice Hall.

ONU. (2015). Objetivos de Desarrollo del Milenio, informe de 2015. New York: ONU.

Russonello, G. (30 de Nov de 2015). Two-thirds os Americans want U.S. to join Climate Change Pact. The New York Times.

Shiva, V. (1997). Biopiracy: the plunder of nature and knowledge. Boston: South end press. 